\title{
Articulatory Suppression Effects on Induced Rumination
}

\author{
Ladislas Nalborczyk ${ }^{1}{ }^{2}$, Marcela Perrone-Bertolotti' $\odot$, Céline Baeyens ${ }^{2} \odot$, Romain Grandchamp ${ }^{1} \odot$, Elsa Spinelli', \\ Ernst H. W. Koster ${ }^{3} \oplus$, Hélène Lœvenbruck ${ }^{1}$ \\ 1 Univ. Grenoble Alpes, Univ. Savoie Mont Blanc, CNRS, LPNC, 38000 Grenoble, France, 2 LIP/PC2S, Univ. Grenoble Alpes, CNRS, Grenoble, France, 3 \\ Department of Experimental Clinical and Health Psychology, Ghent University, Ghent, Belgium \\ Keywords: finger-tapping, articulatory suppression, inner speech, rumination \\ https://doi.org/10.1525/collabra.31051
}

Collabra: Psychology

Vol. 8, Issue 1, 2022

This study explores whether the speech motor system is involved in verbal rumination, a particular kind of inner speech. The motor simulation hypothesis considers inner speech as an action, accompanied by simulated speech percepts, that would as such involve the speech motor system. If so, we could expect verbal rumination to be disrupted by concurrent involvement of the speech apparatus. We recruited 106 healthy adults and measured their self-reported level of rumination before and after a rumination induction, as well as after five minutes of a subsequent motor task (either an articulatory suppression -silent mouthing- task or a finger tapping control task). We also evaluated to what extent ruminative thoughts were experienced with a verbal quality or in another modality (e.g., visual images, non-speech sounds). Self-reported levels of rumination showed a decrease after both motor activities (silent mouthing and finger-tapping), with only a slightly stronger decrease after the articulatory suppression than the control task. The rumination level decrease was not moderated by the modality of the ruminative thoughts. We discuss these results within the framework of verbal rumination as simulated speech and suggest alternative ways to test the engagement of the speech motor system in verbal rumination. Pre-registered protocol, preprint, data, as well as reproducible code and figures are available at: https://osf.io/3bh67/.

\section{Introduction}

A large part of our inner conscious experience involves verbal content, with internal monologues and conversations. Inner speech is considered as a major component of conscious experience and cognition (Hubbard, 2010; Hurlburt et al., 2013; Klinger \& Cox, 1987). An important issue concerns the format and nature of inner speech and whether it is better described as a mere evocation of abstract amodal verbal representations (i.e., without articulatory or auditory sensation) or as a concrete motor simulation of actual speech production (for reviews, see Alderson-Day \& Fernyhough, 2015; Lœvenbruck et al., 2018; Perrone-Bertolotti et al., 2014). In the first case, inner speech is seen as divorced from bodily experience, and includes, at most, faded auditory representations. In the second case, inner speech is considered as a physical process that unfolds over time, leading to an enactive re-creation of auditory percepts, via the simulation of articulatory actions. The latter hypothesis is interesting in the context of persistent negative and maladaptive forms of inner speech, such as rumination. If this hypothesis is correct, we could expect rumination -as a particular type of inner speech- to be disrupted by concurrent involvement of the speech muscles. The present study aims at testing this specific idea.

Introspective explorations of the characteristics of inner speech have led to different views on the relative importance of its auditory and articulatory components, and on the involvement of motor processes. It has been suggested successively that speech motor representations would be purely motoric (Stricker, 1880), that they would be expressed dominantly in an auditory format (Egger, 1881), or that they would consist in a mix of these in the overall population (Ballet, 1886). The intuitive distinction between auditory and motor phenomena is sometimes referred to in contemporary research by the terms of inner ear and inner voice, in line with Baddeley's classic model of working memory (e.g., Baddeley et al., 1984; see also Buchsbaum, 2013). Baddeley's model relies on a partnership between an inner ear (i.e., storage) and an inner voice (i.e., subvocal rehearsal), which can be assessed by selectively blocking either one of these components (e.g., Smith et al., 1995).

Empirical arguments supporting the crucial role of the inner voice in verbal working memory (subvocal articulatory rehearsal) can be found in studies using articulatory suppression, in which the action component (i.e., the inner 
voice) of inner speech is disrupted. Articulatory suppression usually refers to a task which requires participants to utter speech sounds (or to produce speech gestures without sound), so that this activity disrupts ongoing speech production processes. Articulatory suppression can be produced with different degrees of vocalisation, going from overt uttering to whispering, mouthing (i.e., silent articulation), and simple clamping of the speech articulators. Many studies have shown that articulatory suppression can be used to disrupt the subvocal rehearsal mechanism of verbal working memory and -as a consequence-impair the recall of verbal material (e.g., Baddeley et al., 1984; Larsen \& Baddeley, 2003).

Based on the study of errors accompanying the covert production of tongue twisters, inner speech has also been suggested to be impoverished (as compared to overt speech) and to lack a full specification of articulatory features (e.g., Oppenheim \& Dell, 2008, 2010). More precisely, these studies have shown the phonemic similarity effect (the tendency, in overt speech, to exchange phonemes with similar articulatory features) to be absent in inner speech. In contrast to these results, however, Corley et al. (2011) found the phonemic similarity effect to be present in inner speech, suggesting that inner speech is not necessarily impoverished at the articulatory level.

In a study aiming at investigating the role of covert enactment in auditory imagery (defined as imagined speech, produced by oneself or another individual), Reisberg et al. (1989) observed that the verbal transformation effect (Warren \& Gregory, 1958), namely the alteration of speech percepts when certain speech sounds are uttered in a repetitive way, also occurred during inner speech (although the verbal transformation effect was smaller than during overt speech), but was suppressed by concurrent articulation (e.g., chewing) or clamping the articulators. The fact that the verbal transformation effect was observed during inner speech and that it was reduced by concurrent chewing, even in inner speech, speaks in favour of the view of inner speech as an enacted simulation of overt speech.

Another piece of evidence for the effect of articulatory suppression on inner speech comes from a recent study by Topolinski \& Strack (2009) on the mere exposure effect, namely the fact that repeated exposure to a stimulus influences the evaluation of this stimulus in a positive way (Zajonc, 1968). Topolinski and Strack's study showed that the mere exposure effect for visually presented verbal material could be completely suppressed by blocking subvocal rehearsal (i.e., inner speech) when asking participants to chew a gum. The effect was preserved, however, when participants kneaded a soft ball with their hand (Topolinski \& Strack, 2009). This finding suggests that blocking speech motor simulation interfered with the inner rehearsal of the visually presented verbal stimuli, thereby destroying the positive exposure effect. It provides additional experimental support to the view that inner speech involves a motor component.

Articulatory suppression effects have usually been found to be weaker in covert than overt speech production, however. Although this can be interpreted as a lesser role for articulatory processes in covert mode, it does not fully rule out articulatory involvement. In overt speech, various de- grees of articulatory monitoring can be observed, as explained in the Hyper \& Hypo-speech theory (Lindblom, 1990). In casual, or hypo-, speech, articulatory targets are often missed, whereas in more demanding situation, or hyper-speech, more controlled articulatory gestures may be observed. A less stringent articulatory control does not preclude speech production, even in the overt mode. The fact that a few studies have found subphonemic effects in covert mode (e.g., Corley et al., 2011) and that articulatory features are so much at play that they may even capture simultaneous speech perception (Scott et al., 2013) does suggest that articulatory processes are, at least sometimes, recruited. Furthermore, various degrees of articulatory expansion and involvement may be expected in various forms of inner speech, depending on cognitive demands, as described in Grandchamp et al. (2019). Altogether, these findings suggest that some variants of inner speech may be associated with speech motor processes.

The occurrence of motor simulation during inner speech is further backed by several studies using physiological measures to evaluate inner speech production properties. Using electrodes inserted in the tongue tip or lips of five participants, Jacobson (1931) was able to detect electromyographic (EMG) activity during several tasks requiring inner speech. Similarly, Sokolov (1972) recorded intense lip and tongue muscle activation when participants had to perform complex tasks that necessitated substantial inner speech production (e.g., problem solving). Another study using surface electromyography (sEMG) demonstrated an increase in activity of the lip muscles during silent recitation tasks compared to rest, but no increase during the nonlinguistic visualisation task (Livesay et al., 1996). An increase in the lip and forehead muscular activity has also been observed during induced rumination (Nalborczyk et al., 2017). Furthermore, this last study also suggested that speech-related muscle relaxation was slightly more efficient in reducing subjective levels of rumination than non speech-related muscle relaxation, suggesting that relaxing or inhibiting the speech muscles could disrupt rumination.

Rumination is a "class of conscious thoughts that revolve around a common instrumental theme and that recur in the absence of immediate environmental demands requiring the thoughts" (Martin \& Tesser, 1996). Despite the fact that depressed patients report positive metacognitive beliefs about ruminating, which is often seen as a coping strategy in order to regulate mood (e.g., Papageorgiou \& Wells, 2001), rumination is known to significantly worsen mood (e.g., Moberly \& Watkins, 2008; Nolen-Hoeksema \& Morrow, 1993), impair cognitive flexibility (e.g., Davis \& Nolen-Hoeksema, 2000; Lyubomirsky et al., 1998), and to lead toward pronounced social exclusion and more interpersonal distress (Lam et al., 2003). Although partly visual, rumination is a predominantly verbal process (Goldwin \& Behar, 2012; McLaughlin et al., 2007) and can therefore be considered as a maladaptive type of inner speech. In a study on worry, another form of repetitive negative thinking, Rapee (1993) observed a tendency for articulatory suppression, but not for visuo-spatial tasks, to produce some interference with worrying. He concluded that worry involves the phonological aspect of the central executive of working memory. We further add that, since repeating a 
word seems to reduce the ability to worry, this study suggests that articulatory aspects are at play during worry.

In this context, the question we addressed in this study is whether verbal rumination consists of purely abstract verbal representations or whether it is better described as a motor simulation of speech production, engaging the speech apparatus. If the latter hypothesis is correct, rumination experienced in verbal form (in contrast to other forms, such as pictoral representations) should be disrupted by mouthing (i.e., silent articulation), and should not be disrupted by a control task that does not involve speech muscles (e.g., finger-tapping). Specifically, we thus sought to test two hypotheses. First, we tested whether rumination could be disrupted by articulatory suppression but not by finger-tapping, by examining the interaction between session and motor task. Second, we tested whether this disruption would be more pronounced when rumination is experienced in a verbal form than in a non-verbal form.

\section{Methods}

In the Methods and Data analysis sections, we report how we determined our sample size, all data exclusions, all manipulations, and all measures in the study (Simmons et al., 2012). A pre-registered version of our protocol can be found on OSF: https://osf.io/3bh67/.

\section{Participants}

We originally planned for 128 participants to take part in the study. This sample size was set on the basis of results obtained by Topolinski \& Strack (2009), who observed an effect size around $\eta_{p}^{2}=.06$. We expected a similar effect size for the current rumination disruption, since rumination can be conceived of as a subtype of inner speech. ${ }^{1}$

As we anticipated drop-out of participants due to our inclusion criteria (see below), a total of 184 undergraduate students in psychology from Univ. Grenoble Alpes took part in this experiment, in exchange for course credits. They were recruited via mailing list, online student groups, and posters. Each participant provided a written consent and this study was approved by the local ethics committee (CERNI N²016-05-31-9). To be eligible, participants had to be between 18 and 35 years of age, with no self-reported history of motor, neurological, psychiatric, or spoken language disorders. All participants spoke French as their mother tongue. After each participant gave their written consent, they completed the Center for Epidemiologic Studies - Depression scale (CES-D, Radloff, 1977). The CES-D is a 12-item questionnaire, validated in French (Morin et al., 2011), aiming to assess the level of depressive symptoms in a subclinical population. Participants exceeding the threshold of clinical depressive symptoms (i.e., >23 for females and $>17$ for males, Radloff, 1977) were not included in the study for ethical reasons $(\mathrm{N}=26)$. These participants were then fully debriefed about the aims of the experiment and were given the necessary information concerning available psychological care on campus.

To investigate the influence of articulatory suppression on induced rumination in healthy participants, a successful induction of rumination is a prerequisite. Examination of the data showed that not all participants reported an increase in state rumination after induction. Therefore, the inclusion criteria diverted from the preregistration. Analyses were only conducted on participants who reported more rumination after the induction than before. We thus discarded participants who did not show any increase in rumination level ( $\mathrm{N}=52,32.91 \%$ of total sample). ${ }^{2}$ The final sample comprised 106 participants (Mean age $=20.30, \mathrm{SD}=$ 2.57, Min-Max = 18-31, 96 females).

\section{Material}

The experiment was programmed with OpenSesame software (Mathôt et al., 2012) and stimuli were displayed on a DELL latitude E6500 computer screen.

State affect (PANAS). To control for confounding variables likely to be related to the intensity of the induction procedure, we administered the French version of the Positive and Negative Affect Schedule (PANAS, Watson et al., 1988), adapted to French by Gaudreau et al. (2006). This questionnaire includes 20 items, from which we can compute an overall index of both positive (by summing the scores on 10 positive items, thereafter PANASpos) and negative affect (PANASneg) at baseline. This questionnaire was administered at baseline.

Trait rumination (RRS). To evaluate trait rumination, participants completed the short version of the Ruminative Response Scale (RRS-R, Treynor et al., 2003) at the end of the experiment. From this questionnaire (validated in French; Guimpel et al., 2012), scores on two dimensions were analysed (RRSbrooding and RRSreflection).

State rumination measures. Measures of state rumination were performed using a Visual Analogue Scale (VAS) previously used and justified in Nalborczyk et al. (2017). This scale measured the degree of agreement with the sentence "At this moment, I am brooding on negative things" (translated from French), on a continuum between "Not at all" and "A lot" (afterwards coded between 0 and 100). This one-item rating was specifically designed to minimise complicated metacognitive judgment and to shorten assessment, relative to other rumination measures. This scale is subsequently referred to as the RUM scale. It was used three times in the experiment, at baseline (after training but before the experiment started), after rumination induction, and after a motor task. This repeated brief assessment is very similar to the experience sampling methodology used

\footnotetext{
1 In the original power calculations included in the OSF preregistration platform, we had inadequately specified the effect size in GPower, but we only realised this erroneous specification after the freezing of the preregistration on the OSF platform. Therefore, the current sample size slightly differs from the preregistered one.

2 Analyses performed on the whole sample of participants are presented in the supplementary materials and do not show substantial variations from the analyses presented in the present article.
} 
in Moberly \& Watkins (2008).

Modality of the rumination. Additionally, participants answered questions about the modality of the thoughts that occurred while performing the motor task. This last questionnaire consisted of one question evaluating the occurrence frequency of different modalities of inner thoughts (e.g., visual imagery, verbal thoughts, music). Then, a ver$\mathrm{bal} /$ non-verbal ratio (i.e., the score on the verbal item divided by the mean of the score on the non-verbal items) was computed, hereafter referred to as the Verbality continuous predictor (this scale is available in the online supplementary materials).

Articulatory suppression task. In the first part of the experiment, ruminative thoughts were induced using a classical induction procedure (Nolen-Hoeksema \& Morrow, 1993). Then a motor task was executed. Participants were randomly allocated to one of two groups. In the Mouthing group, the task consisted of repetitively making mouth opening-closing movements at a comfortable pace. This task was selected as it is commonly used in articulatory suppression studies. As a control, a finger-tapping task was used (the Tapping group), that consisted of tapping on the desk with the index finger of the dominant hand at a comfortable pace.

Although finger-tapping tasks are generally considered as good control tasks when using speech motor tasks, since they are comparable in terms of general attentional demands, it may be that orofacial gestures are intrinsically more complex than manual gestures (i.e., more costly, Emerson \& Miyake, 2003). To discard the possibility that orofacial gestures (related to the Mouthing group) would be cognitively more demanding than manual ones (related to the Tapping group), we designed a pre-test experiment in order to compare the two interference motor tasks used in the main experiment. Results of this control experiment showed no difference on reaction times during a visual search task between the two interference tasks (i.e., mouthing and finger-tapping). Full details are provided in the supplementary materials.

\section{Procedure}

The experiment took place individually in a quiet and dimmed room. The total duration of the session ranged between $35 \mathrm{~min}$ and $40 \mathrm{~min}$. Before starting the experiment, participants were asked to perform the motor task during $1 \mathrm{~min}$, while following a dot moving at a random pace on the screen in front of them. This task was designed to train the participants to perform the motor task adequately. Following this training and after describing the experiment, the experimenter left the room and each participant had to fill-in a baseline questionnaire (adaptation of PANAS, see above) presented on the computer screen. Baseline state rumination was then evaluated using the $R U M$ scale. The whole experiment was video-monitored using a Sony HDRCX240E video camera, in order to check that the participants effectively completed the task.

Rumination induction. Rumination induction consisted of two steps. The first step consisted of inducing a negative mood in order to enhance the effects of the subsequent rumination induction. Participants were asked to

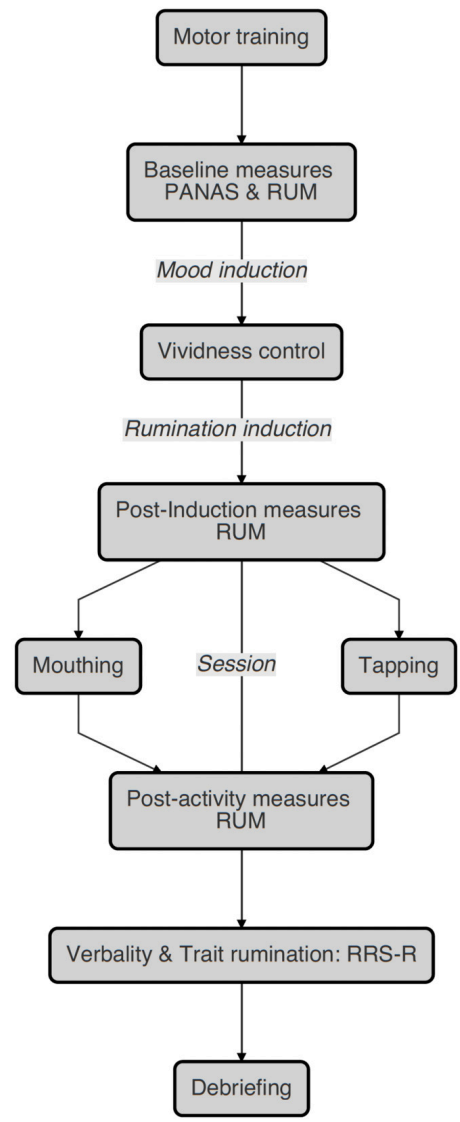
Figure 1. Timeline of the experiment, from top to
bottom.

recall a significant personal failure experienced in the past five years. Then, participants were invited to evaluate the extent to which this memory was "intense for them" on a VAS between "Not at all" and "A lot," afterwards coded between 0 and 100, and referred to as Vividness.

The second step consisted of the rumination induction. We used a French translation of Nolen-Hoeksema \& Morrow (1993)'s rumination induction procedure. Participants had to read a list of 44 sentences related to the meaning, the causes and the consequences of their current affective or physiological state. Each phrase was presented on a computer screen for 10 seconds and the total duration of this step was 7 minutes and 20 seconds. State rumination was then evaluated again using the same VAS as the one used at baseline (RUM).

Motor task. After the rumination induction, participants were asked to continue to think about "the meaning, causes, and consequences" of their feelings while either repetitively making mouth movements (for participants allocated in the "Mouthing" group) or finger-tapping with the dominant hand for five minutes (for participants allocated in the "Tapping" group). Afterwards, state rumination was again evaluated using the RUM scale.

In order to evaluate trait rumination, participants completed the short version of the RRS (see above). Then, they filled in the questionnaire on the modality of the thoughts that occurred while performing the motor task (see above). Figure 1 summarises the full procedure. 


\section{Data analysis}

Statistical analyses were conducted using R version 3.5.3 (R Core Team, 2018) and are reported with the papaja (Aust \& Barth, 2018) and knitr (Xie, 2015) packages.

We centered and standardised each predictor in order to facilitate the interpretation of parameters. To assess the effects of articulatory suppression on self-reported state rumination, data were then analysed using Session (2 levels, before and after motor activity, sum contrasts) as a withinsubject categorical predictor, and Group (2 levels, Mouthing and Tapping) as a between-subject categorical predictor and $R U M$ as a dependent variable in a Bayesian multilevel linear model, using the brms package (Bürkner, 2017). ${ }^{3}$ This model was subsequently compared with more complex models including effects of control variables, including baseline affect state (PANAS scores), trait rumination (RRS scores), the vividness of the memory chosen during the induction (Vividness score), or the degree of verbality of the ruminative thoughts (Verbality index).

Models were compared using the Widely Applicable Information Criterion (WAIC, Watanabe, 2010) -a generalisation of the Akaike information criterion (Akaike, 1974)- and evidence ratios (Burnham et al., 2011; Burnham \& Anderson, 2002; Hegyi \& Garamszegi, 2011). The WAIC provides a relative measure of predictive accuracy of the models (the WAIC is an approximation of the out-of-sample deviance of a model) and balances underfitting and overfitting by sanctioning models for their number of parameters. Evidence ratios (ERs) were computed as the ratios of weights: $E R_{i j}=\frac{w_{i}}{w_{j}}$, where $w_{i}$ and $w_{j}$ are the Akaike weights of models $i$ and $j$, respectively. These weights can be interpreted as the probability of the model being the best model in terms of out-of-sample prediction (Burnham \& Anderson, 2002). Whereas the use of WAIC is appropriate for model comparison and selection, it tells us nothing about the absolute fit of the model. To estimate this fit, we computed the Bayesian $R^{2}$ for MLMs using the bayes_R2() method in the brms package (Bürkner, 2017).

Models were fitted using weakly informative priors (see the supplementary materials for code details). Two Markov Chain Monte-Carlo (MCMC) were ran for each model to approximate the posterior distribution, including each 5.000 iterations and a warmup of 2.000 iterations. Posterior convergence was assessed examining trace plots as well as the Gelman-Rubin statistic $\hat{R}$. Constant effect estimates were summarised via their posterior mean and $95 \%$ credible interval (CrI), where a credible interval can be considered as the Bayesian analogue of a classical confidence interval. When applicable, we also report Bayes factors (BFs), computed using the Savage-Dickey method, which consists in taking the ratio of the posterior density at the point of interest divided by the prior density at that point. These BFs can be interpreted as an updating factor, from prior knowl- edge (what we knew before seeing the data) to posterior knowledge (what we know after seeing the data).

\section{Results}

The Results section is divided into two parts. First, we present results from confirmatory analyses, aiming to assess the effects of articulatory suppression (as compared to finger-tapping) on self-reported state rumination. Second, we present results from exploratory analyses, aiming to assess the role of moderator variables such as baseline state affects or trait rumination. Moreover, the Results section follows the data analysis workflow. More precisely, we first present the results of the model comparison stage in which we compare different models of increasing complexity. Subsequently, we report the estimates of the best model (i.e., the model with the lowest WAIC) on which we base our conclusions.

\section{Descriptive statistics}

Recall that, in assessing the effects of articulatory suppression, the dependent variable is $R U M$, the main categorical predictors are Session (within-subject) and Group (between-subject), and additional continuous predictors are PANAS, RRS and Vividness. Summary statistics (mean and standard deviation) for all these variables can be found in Table 1.

Figure 2 shows the overall evolution of the mean RUM rating (i.e., self-reported state rumination) through the experiment according to each Session (Baseline, Post-induction, Post-motor) and Group (Mouthing, Tapping). As displayed in this figure, important inter-individual variability was observed in all conditions. After the rumination induction, RUM increased in both groups, and decreased after the motor task, with a slightly stronger decrease in the Mouthing group.

\section{Confirmatory analyses}

To examine the effect of the two motor tasks (articulatory suppression and finger-tapping, Group variable) on RUM while controlling for other variables (i.e., Vividness, RRSbrooding, RRSreflection, Verbality, PANASpos, and $P A N A S n e g)$, we compared several models in accordance with our preregistered plans. More precisely, we compared models including only the effect of Group, only the effect of Session, both simple effects and the interaction between Group and Session and a model adding Verbality as a control variabe. We expected that greater amounts of verbal thoughts would be associated with a greater difference in the effects of the motor task on self-reported state rumination (i.e., $R U M$ ) with respect to the group (i.e., mouthing vs. fingertapping).

Model comparison showed that the best model was the model including Session, Group, an interaction term be- 
Table 1. Descriptive statistics (mean and standard deviation) of each recorded variable, for the final sample of participants that were included in the study.

\begin{tabular}{|c|c|c|c|c|c|c|}
\hline \multirow[b]{2}{*}{ Variables } & \multicolumn{3}{|c|}{ Mouthing } & \multicolumn{3}{|c|}{ Tapping } \\
\hline & Baseline & $\begin{array}{l}\text { Post- } \\
\text { induction }\end{array}$ & Post-motor & Baseline & $\begin{array}{c}\text { Post- } \\
\text { induction }\end{array}$ & Post-motor \\
\hline RUM & $\begin{array}{c}28.5 \\
(26.49)\end{array}$ & $\begin{array}{c}54.66 \\
(25.16)\end{array}$ & $\begin{array}{c}45.47 \\
(27.25)\end{array}$ & $\begin{array}{c}20.96 \\
(21.82)\end{array}$ & $\begin{array}{c}46.77 \\
(25.74)\end{array}$ & $\begin{array}{c}43.54 \\
(29.57)\end{array}$ \\
\hline Age & $20.3(2.65)$ & - & - & $\begin{array}{l}20.31 \\
(2.53)\end{array}$ & - & - \\
\hline PANASneg & $\begin{array}{l}15.65 \\
(5.67)\end{array}$ & - & - & $\begin{array}{l}15.46 \\
(5.08)\end{array}$ & - & - \\
\hline PANASpos & $\begin{array}{l}30.91 \\
(4.48)\end{array}$ & - & - & $31.25(4.4)$ & - & - \\
\hline RRSbrooding & $12.2(2.43)$ & - & - & $\begin{array}{l}12.06 \\
(2.62)\end{array}$ & - & - \\
\hline RRSreflection & $\begin{array}{l}12.22 \\
(3.22)\end{array}$ & - & - & $\begin{array}{l}11.71 \\
(3.26)\end{array}$ & - & - \\
\hline Verbality & $1.67(1.18)$ & - & - & $1.67(1.26)$ & - & - \\
\hline Vividness & $\begin{array}{c}54.17 \\
(28.94)\end{array}$ & - & - & $\begin{array}{c}59.78 \\
(24.63)\end{array}$ & - & - \\
\hline
\end{tabular}

tween Session and Group, and Verbality as predictors (cf. Table 2). Absolute fit of the best model was moderate $\left(R^{2}=\right.$ $0.653,95 \%$ CrI [0.559, 0.722]).

Parameter values of the best model are reported in Table $\underline{3}$. Based on these values, it seems that self-reported rumination decreased after both motor tasks (the coefficient for Session is negative), but this decrease was substantially larger in the Mouthing group $\left(d_{a v}=-0.35,95 \%\right.$ CI $[-0.74$, 0.03]) than in the Tapping group $\left(d_{a v}=-0.12,95 \%\right.$ CI [-0.51, $0.27])$, as can be read from the coefficient of the interaction term between Session and Group (Est $=5.01, \mathrm{SE}=4.06,95 \%$ CrI [-2.86, 13.02]). Importantly, the large uncertainty associated with this result (as expressed by the width of the credible interval) warrants a careful interpretation of this result, that should be considered as suggestive evidence, rather than conclusive evidence.

However, the Bayesian framework provides tools that permit richer inference. First, we can look at the BF for this particular parameter. As can be seen from Table 3 , the $\mathrm{BF}_{10}$ for the interaction term is equal to 0.83 , which is evidence for neither the presence or the absence of effect. However, this BF is computed using the Savage-Dickey method ${ }^{4}$ and as such is extremely sensitive to the prior choice. Thus, other priors (for instance a prior that is more peaked on zero) could provide stronger evidence for the interaction effect.

Second, and more interestingly, we can also directly look at the posterior distribution of the parameter of interest (the interaction term). This reveals that although the 95\% credible interval largely encompasses 0 , there is a 0.89 probability that the interaction between Session and Group

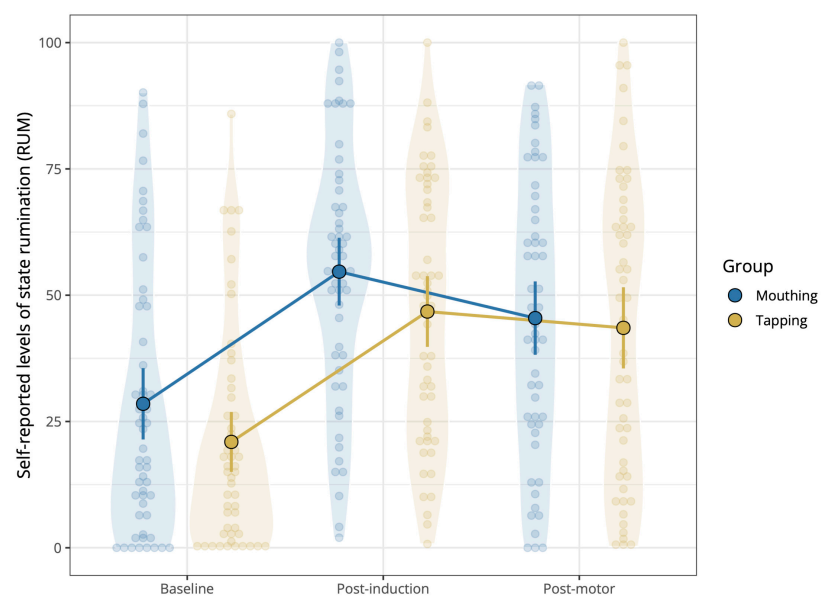

Figure 2. Mean RUM rating by Session and Group, along with violin plots and individual data. Error bars represent $95 \%$ confidence intervals.

is positive (given the data and the priors).

Finally, the large variation between participants can be appreciated by computing the intra-class correlation (ICC), expressed as $\sigma_{\text {intercept }}^{2} /\left(\sigma_{\text {intercept }}^{2}+\sigma_{\text {residuals }}^{2}\right)$. For the best model, the ICC is equal to 0.62 (95\% CrI [0.45, 0.73]), indicating that $61.50 \%$ of the variance in the outcome that remains after accounting for the effect of the predictors, is attributable to systematic inter-individual differences.

Figure 3 shows the effects of Verbality on the relative

\footnotetext{
4 This method simply consists in taking the ratio of the posterior density at the point of interest divided by the prior density at that point (for a practical introduction, see Wagenmakers et al., 2010).
} 
Table 2. Comparison of models, ordered by WAIC. The best model has the lowest WAIC.

\begin{tabular}{lcccc}
\hline & WAIC & pWAIC & $\Delta_{\text {WAIC }}$ & Weight \\
\hline Session + Group + Session : Group + Verbality & 1864.50 & 69.53 & 0.000 & 0.38 \\
Session + Group + Session : Group & 1864.66 & 69.28 & 0.151 & 0.35 \\
Session & 1865.21 & 68.40 & 0.703 & 0.27 \\
Null model & 1877.64 & 67.48 & 13.132 & 0.00 \\
Group & 1878.17 & 67.41 & 13.662 & 0.00 \\
\hline
\end{tabular}

Note. All models include a constant intercept and a varying intercept for Participant.

Table 3. Coefficient estimates, standard errors (SE), 95\% CrI (Lower, Upper), Rhat and Bayes factor (BF10) for the best model.

\begin{tabular}{lcccccc}
\hline Term & Estimate & SE & Lower & Upper & Rhat & BF10 \\
\hline Intercept & 47.630 & 2.321 & 43.038 & 52.140 & 1.003 & $7.104^{*} 10^{\wedge} 14$ \\
Session & -5.933 & 2.174 & -10.202 & -1.787 & 1.001 & 9.523 \\
Group & -4.173 & 4.357 & -12.805 & 4.155 & 1.000 & 0.687 \\
Verbality & 2.165 & 2.341 & -2.394 & 6.727 & 1.000 & 0.355 \\
Session:Group & 5.011 & 4.060 & -2.860 & 13.015 & 1.001 & 0.832 \\
\hline
\end{tabular}

change (i.e., after - before) in self-reported rumination after both motor activities (i.e., Mouthing and Tapping). As Verbality was centred before analysis, its score cannot be interpreted in absolute terms. However, a high score on this index indicates more verbal than non-verbal (e.g., visual images, non-speech sounds) thoughts, whereas a low score indicates more non-verbal than verbal thoughts. Contrary to our predictions but consistent with the model comparison, this figure depicts a similar relationship between Verbality and the change in RUM score (between before and after the motor task), according to the Group. In the Mouthing group, the change in RUM score did decrease for participants with a higher self-reported degree of verbal content. This suggests that the more verbal the rumination is, the more it is affected by mouthing interference. But contrary to our expectation, a similar trend (although perhaps weaker) was observed in the Tapping group. This suggests that the more verbal the rumination is, the more it is affected by any motor task.

\section{Exploratory analyses}

To examine the effect of the two motor tasks (articulatory suppression and finger-tapping, Group variable) on $R U M$ while controlling for other variables (i.e., Vividness, RRSbrooding, RRSreflection, Verbality, PANASpos, and PANASneg), we then compared the best model identified in the previous section with several other models including these variables.

This analysis revealed that the best model (again, the model having the lowest WAIC) was the "full model," that

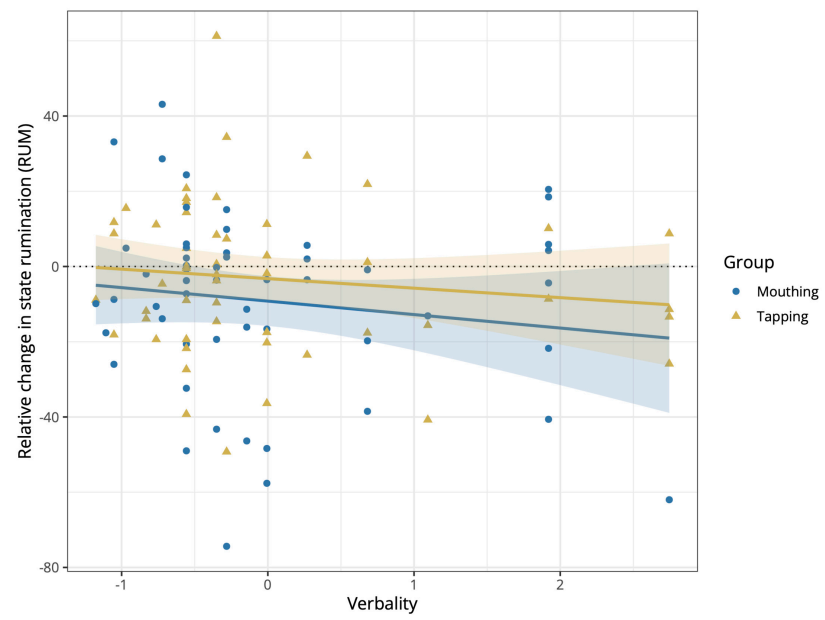

Figure 3. Mean RUM relative change after motor activity, as a function of the degree of Verbality, in the mouthing (the blue dots and regression line) and finger tapping (the yellow triangles and regression line) groups.

is, the model including Session, Group, an interaction between Session and Group, Verbality, PANASpos, PANASneg, RRSbrooding, RRSreflection, and Vividness (cf. Table 4). Absolute fit of the best model was moderate $\left(R^{2}=0.657\right.$, 95\% CrI $[0.566,0.725])$. 
Table 4. Comparison of models, ordered by WAIC. The best model has the lowest WAIC.

\begin{tabular}{|c|c|c|c|c|}
\hline & $W A I C$ & $p W A I C$ & $\Delta_{W A I C}$ & Weight \\
\hline Session + Group + Session $:$ Group + Verb + PANASp + PANASs $+R R S r+R R S b+V i v$ & 1861.12 & 66.70 & 0.000 & 0.38 \\
\hline Session + Group + Session $:$ Group + Verb + Viv & 1862.19 & 67.95 & 1.072 & 0.23 \\
\hline Session + Group + Session $:$ Group + Verb + RRSb & 1863.18 & 68.54 & 2.057 & 0.14 \\
\hline Session + Group + Session $:$ Group + Verb + PANASp & 1863.87 & 68.73 & 2.745 & 0.10 \\
\hline Session + Group + Session $:$ Group + Verb & 1864.50 & 69.53 & 3.382 & 0.07 \\
\hline Session + Group + Session $:$ Group + Verb + PANASn & 1864.88 & 69.11 & 3.761 & 0.06 \\
\hline Session + Group + Session $:$ Group + Verb $+R R S r$ & 1866.53 & 70.02 & 5.409 & 0.03 \\
\hline
\end{tabular}

Note. All models include a constant intercept and a varying intercept for Participant. $V e r b=$ Verbality, $V i v=$ Vividness, $P A N A S p=$ PANAS positive affects, $P A N A S n=$ PANAS negative affects, $R R S b=$ RRSbrooding, $R R S r=$ RRSreflection. 
Table 5. Coefficient estimates, standard errors (SE), 95\% CrI (Lower, Upper), Rhat and Bayes factor (BF10) for the best model.

\begin{tabular}{lcccccc}
\hline Term & Estimate & SE & Lower & Upper & Rhat & BF10 \\
\hline Intercept & 47.607 & 2.096 & 43.562 & 51.749 & 1.000 & $1.008^{*} 10^{\wedge} 15$ \\
Session & -5.899 & 2.140 & -10.093 & -1.783 & 1.000 & 7.857 \\
Group & -5.059 & 3.900 & -12.689 & 2.487 & 1.001 & 0.93 \\
Verbality & 3.236 & 2.090 & -0.899 & 7.314 & 1.001 & 0.694 \\
PANASpos & -6.508 & 2.115 & -10.676 & -2.285 & 1.000 & 16.13 \\
PANASneg & 4.234 & 2.227 & -0.193 & 8.539 & 1.000 & 1.209 \\
RRSreflection & -1.342 & 2.098 & -5.540 & 2.807 & 1.000 & 0.256 \\
RRSbrooding & 1.658 & 2.250 & -2.714 & 6.215 & 1.001 & 0.296 \\
Vividness & 7.560 & 2.168 & 3.270 & 11.755 & 1.000 & 51.81 \\
Session:Group & 5.015 & 3.992 & -2.714 & 12.724 & 1.000 & 0.831 \\
\hline
\end{tabular}

Parameter values of the best model are reported in Table $\underline{5}$. Based on these values, it seems that Vividness had a strong positive influence on self-reported state rumination, as can be read from the regression coefficient (Est $=7.56$, $\left.\mathrm{SE}=2.17,95 \% \mathrm{CrI}[3.27,11.76], \mathrm{BF}_{10}=51.81\right)$. Conversely, the summed score on the positive affects dimension of the PANAS (PANASpos) had a protective effects with regards to state rumination, with more positive affects at baseline being associated with lower levels of self-reported state rumination $\left(\mathrm{Est}=-6.51, \mathrm{SE}=2.11,95 \% \mathrm{CrI}[-10.68,-2.29], \mathrm{BF}_{10}\right.$ $=16.13)$.

\section{Discussion}

The purpose of the current study was to investigate the effects of articulatory suppression on induced verbal rumination. We predicted that if verbal rumination, which can be construed as a type of inner speech, does involve the mental simulation of overt speech production, its generation should be disrupted by articulatory suppression, but not by finger tapping. This prediction was not strictly corroborated by the data, as we observed a decrease of self-reported rumination after both types of motor activities (cf. Figure 2 and Table 2), with a somewhat stronger decrease in the Mouthing group. In the following, we examine the validity of our methods and discuss interpretations of our results. Finally, we formulate how subsequent research should address this kind of question and suggest alternative ways to test the above mentioned hypothesis. We begin by discussing the results of the rumination induction procedure.

\section{Rumination induction}

It is noteworthy that $32.91 \%$ of the total sample of participants who were recruited did not respond to this induction, and were therefore not included in the analyses. Moreover, as reported in Table 5, it seems that the Vividness of the memory chosen by the participant during the mood induction was influencing the level of self-reported state rumination. In other words, more vivid (i.e., more "intense") memories were associated with higher levels of self-re- ported state rumination. This highlights the fact that this aspect should be carefully controlled each time a mood induction is used in order to foster subsequent repetitive negative thinking.

Moreover, we observed a group difference of approximately 7.5 points in the average $R U M$ score at baseline. This difference might be explained by motor training, which took place before baseline measurement of state rumination. During this training, participants had to perform the motor task (either finger-tapping or mouthing) in front of a black screen on which a white dot was moving randomly for $1 \mathrm{~min}$. During the task, the experimenter stayed in the room to check that participants were performing the motor task adequately. Being an unusual and potentially embarrassing motor activity, mouthing might have been a higher source of stress for the participants, as compared to the more common activity of finger-tapping. This group difference in baseline state rumination subsisted after the induction, as the group difference after the induction was of approximately 8 points (see summary statistics in Table 1 and full dataset in the supplementary materials). Although the experimenter was present in the training phase to check that instructions were well understood, which might have increased baseline levels of rumination, participants were left alone in the subsequent phases, therefore the embarrassment feeling was not as strong.

\section{Articulatory suppression effects}

In the following section, we discuss in more depth the results of the second part of the study, which aimed at comparing the effects of articulatory suppression and fingertapping on self-reported rumination.

First, it is important to examine whether the weakness of the effect of the interaction we had predicted between session and group could come from a lack of statistical power. We planned 128 participants in order to reach a power of .80 for a targeted effect size of $\eta_{p}^{2}=.06$. As explained above, out of the 184 recruited participants, only 106 could be included in the study. With 106 participants, the a priori power for 
detecting an effect size of $\eta_{p}^{2}=.06$ was approximately of .70 , which is much higher than the median power in typical psychological studies.

Second, it is important to acknowledge that despite the weakness of the difference between the two groups in their influence on the level of self-reported rumination (i.e., $R U M)$, both activities did lead, on average, to a decrease in self-reported rumination of approximately 6 points on the VAS (as indicated by the slope for Session in Table 3). This decrease might be interpreted in two ways. First, it might be explained by the simple exposition to the VAS and by compliance effects. When asked to rate their level of rumination again after five minutes of motor activity, some participants might be prompted to indicate a lower level of rumination than before the motor task. But compliance effects could similarly lead participants to consider the motor task as irritating, and therefore as prone to rumination increase. Some participants could therefore also be biased towards indicating a higher level of rumination after the motor task. Second, it might be considered that this decrease reflects a genuine decrease in rumination. In the following, we adopt the latter perspective and discuss explanations for the weak difference between the two groups.

Effect of the rumination quality (verbality). Our prediction was that rumination in verbal form would be more disrupted by mouthing than rumination in non-verbal form, while both kinds of rumination would not be disrupted (or similarly disrupted) by finger-tapping. In other words, we hypothesised a three-way interaction, between the effect of time (i.e., Session), Group, and Verbality. In the following, we discuss the absence of this interaction. Then, we focus on the weak difference between the two groups (omitting Verbality), and discuss some explanations for this weak difference.

First, the absence of the three-way interaction might come from a difficulty for the participants to have clear introspective access to the ruminative thoughts they experienced during the motor task. For instance, we know that introspective description of inner speech differs considerably, between people trained to regularly report on their episodes of inner speaking, and people without such training (e.g., Hurlburt et al., 2013). Moreover, as the Verbality questionnaire was presented at the end of the experiment, one cannot exclude that it was partly contaminated by recall, which, when done verbally, has been shown to artificially increase the subjective verbality index (Hurlburt, 2011).

Difference between motor groups. Leaving the self-reported quality of rumination aside, we now turn to a discussion of the weak difference between the two motor groups. We think this result can be explained in at least two nonexclusive ways. First, we could argue that the decrease observed in both groups was due to an unexpected effect of finger-tapping on rumination. Second, we could argue that the effect of the articulatory suppression was somehow weaker than expected. In the following, we provide arguments and explanations for each of these possibilities.

Steady finger-tapping is usually considered as a relevant control condition for evaluating articulatory suppression, since it specifically recruits the hand motor system and should not interfere with the oral motor system, while being comparable in terms of general attentional demands (e.g., Gruber, 2001; Logie \& Baddeley, 1987). However, using more complex rhythmic patterns of finger-tapping, Saito (1994) observed a fade-out of the phonological similarity effect in a verbal memory task with spoken recall, when subjects were asked to tap with either their right (dominant) or left hand, while the phonological similarity effect was conserved in the control condition (no tapping). The author concluded that a complex rhythmic tapping task can interfere with the running of speech motor programs (Saito, 1994, p. 185). More specifically, he suggested that complex, non-automatised, rhythmic finger tapping could use speech motor programs, which are useful to control speech prosody and rhythmic activity. We further suggest that a novel complex rhythmic task might require silent verbalisation and, therefore, might itself be an articulatory suppression task. In line with these findings, another study showed that for right-handed subjects, tapping with a finger of the right hand is more effective at interfering with performance of a verbal memory task than is tapping with a finger of the left hand (Friedman et al., 1988). Although Friedman et al.'s findings are difficult to interpret, because task priority was manipulated and this may have led to conflict resolution, which might have been dealt with differentially according to the hand involved, they do suggest that a finger tapping task is not always the best control for articulatory suppression. This might explain the decrease of self-reported rumination observed in our own study, after the finger-tapping, and suggests that we might observe different results by asking participants to tap with the finger of their non-dominant hand. We think it is important to note for future studies that our results, together with those of Saito (1994) and Friedman et al. (1988), suggest that finger-tapping could in fact interfere with inner speech. In other words, finger-tapping, with the dominant hand, is probably not an appropriate control condition when studying articulatory suppression.

An alternative way to explain the absence of differences between the two motor groups is to suppose that the effects of the articulatory suppression were weaker than we expected. The rhythmic mouthing task might have become too automatised to disrupt inner speech programming. This idea finds some support in the results of Saito (1997), who observed an effect of articulatory suppression on the phonological similarity effect in a memory task only when the articulatory suppression was intermittent (i.e., "ah, ah, ah...”) but no effect when participants had to utter a continuous “ah-." This can be explained by considering that the intermittent articulatory suppression would impose a greater load on speech motor programming than the continuous articulatory suppression (Saito, 1997, p. 569). In a similar vein, Macken \& Jones (1995) found stronger effects of articulatory suppression when participants were asked to repeat a sequence of different letters than when they were asked to repeat a single letter. One way to examine this hypothesis within our own protocol would be to ask participants to make sequences of various mouth movements, rather than repeating a single movement. Alternatively, the relatively weak effects of articulatory suppression on rumination may also be explained by the specific time course of our experimental design. Indeed, the articulatory sup- 
pression was performed after participants went through the entire rumination induction procedure (i.e., after reading all the rumination induction prompts). We speculate that the effects of the articulatory suppression might have been stronger if it had been performed during the rumination (e.g., between each prompt) instead.

In a broader perspective, relating to the original research question, we should mention two additional interpretations of our results. So far, we considered different ways to explain either how the finger-tapping task could interfere with rumination or how the articulatory suppression task might have failed to disrupt rumination. However, if we assume that our scales (especially the RUM outcome response and the Verbality scale) are reliable and that the articulatory suppression was efficient in its intended purpose (i.e., suppressing speech motor activity), we are forced to admit that either i) rumination is not a type of inner speech that can be disrupted by peripheral muscle perturbation (i.e., it could be described as a more abstract form of inner speech) or that ii) inner speech, more broadly, does not depend on peripheral speech muscle activity. Although we think that these questions cannot be answered from our present results, we acknowledge that these two possibilities are compatible with our results.

In summary, the current research is one of the first behavioural studies exploring the association between verbal rumination and the speech motor system. While the observed data did not strictly corroborate our original hypotheses, we explored several explanations for the weak difference between articulatory suppression and the control task, and related our findings to previous works on the role of inner speech in verbal working memory. These results have important implications for future studies on articulatory suppression during inner speech or verbal working memory tasks. More precisely, they highlight the need for further investigation of the most appropriate control task when studying the effects of articulatory suppression.

\section{Supplemental Materials}

Pre-registered protocol, open data, supplementary analyses as well as reproducible code and figures are available at https://osf.io/c9pag/.

A lot of useful packages have been used for the writing of this paper, among which the ggplot2, ggforce, GGally, DiagrammeR, patchwork, BEST, and plotly packages for plotting (Iannone, 2018; Kruschke \& Meredith, 2018; Pedersen, 2017, 2018; Schloerke et al., 2018; Sievert, 2018; Wickham, 2016), the sjstats and tidybayes packages for data analysis (Kay, 2018; Lüdecke, 2018), as well as the tidyverse and glue packages for code writing and formatting (Hester, 2018; Wickham, 2017).

\section{Acknowledgements}

We thank David Meary for his technical support in programming the eye-tracking experiment, Elena Keracheva for her help during data collection as well as Rafael Laboissière and Brice Beffara for their advice regarding data analysis.

\section{Funding Information}

This project was funded by the ANR project INNERSPEECH (grant number ANR-13-BSH2-0003-01). The first author of the manuscript is funded by a doctoral fellowship from Univ. Grenoble Alpes.

\section{Data Accessibility Statement}

Pre-registered protocol, preprint, data, as well as reproducible code and figures are available at: https://osf.io/ 3bh67/.

\section{Author Contributions}

LN, MPB, CB, RG, EK, and HL contributed to conception and design of the study, LN collected the data, LN, MPB, CB, $\mathrm{EK}$, and HL contributed to data analysis and interpretation, LN, MPB, CB, RG, ES, EK, and HL drafted and/or revised the article and all authors approved the submitted version for publication.

\section{Competing Interests}

The authors have no competing interests to declare.

Submitted: March 25, 2021 PST, Accepted: December 06, 2021 PST 


\section{REFERENCES}

Akaike, H. (1974). A new look at the statistical model identification. IEEE Transactions on Automatic Control, 19(6), 716-723. https://doi.org/10.1109/tac.1 974.1100705

Alderson-Day, B., \& Fernyhough, C. (2015). Inner speech: Development, cognitive functions, phenomenology, and neurobiology. Psychological Bulletin, 141(5), 931-965. https://doi.org/10.1037/bul 0000021

Aust, F., \& Barth, M. (2018). papaja: Create APA manuscripts with $R$ Markdown. https://github.com/crs h/papaja

Baddeley, A. D., Lewis, V., \& Vallar, G. (1984). Exploring the articulatory loop. The Quarterly Journal of Experimental Psychology Section A, 36(2), 233-252. htt ps://doi.org/10.1080/14640748408402157

Ballet, G. (1886). Le langage intérieur et les diverses formes de l'aphasie. Alcan.

Buchsbaum, B. R. (2013). The role of consciousness in the phonological loop: Hidden in plain sight. Frontiers in Psychology, 4. https://doi.org/10.3389/fpsy g.2013.00496

Bürkner, P.-C. (2017). brms: An R package for Bayesian multilevel models using Stan. Journal of Statistical Software, 80(1), 1-28. https://doi.org/10.18637/jss.v08 $\underline{0 . \mathrm{i} 01}$

Burnham, K. P., \& Anderson, D. R. (2002). Model selection and multimodel inference: A practical information-theoretic approach (2nd ed.). Springer.

Burnham, K. P., Anderson, D. R., \& Huyvaert, K. P. (2011). AIC model selection and multimodel inference in behavioral ecology: Some background, observations, and comparisons. Behavioral Ecology and Sociobiology, 65(1), 23-35. https://doi.org/10.100 7/s00265-010-1029-6

Corley, M., Brocklehurst, P. H., \& Moat, H. S. (2011). Error biases in inner and overt speech: Evidence from tongue twisters. Journal of Experimental Psychology: Learning, Memory, and Cognition, 37(1), 162-175. http s://doi.org/10.1037/a0021321

Davis, R. N., \& Nolen-Hoeksema, S. (2000). Cognitive Inflexibility Among Ruminators and Nonruminators. Cognitive Therapy and Research, 24(6), 699-711. http s://doi.org/10.1023/a:1005591412406

Egger, V. (1881). La parole intérieure: Essai de psychologie decriptive. Ancienne Librairie Germer Brailliere.

Emerson, M. J., \& Miyake, A. (2003). The role of inner speech in task switching: A dual-task investigation. Journal of Memory and Language, 48(1), 148-168. http s://doi.org/10.1016/s0749-596x(02)00511-9

Friedman, A., Polson, M. C., \& Dafoe, C. G. (1988). Dividing attention between the hands and the head: Performance trade-offs between rapid finger tapping and verbal memory. Journal of Experimental Psychology: Human Perception and Performance, 14(1), 60-68. https://doi.org/10.1037/0096-1523.14.1.60
Gaudreau, P., Sanchez, X., \& Blondin, J.-P. (2006). Positive and negative affective states in a performance-related setting testing the factorial structure of the PANAS across two samples of FrenchCanadian participants. European Journal of Psychological Assessment, 22(4), 240-249. https://do i.org/10.1027/1015-5759.22.4.240

Goldwin, M., \& Behar, E. (2012). Concreteness of idiographic periods of worry and depressive rumination. Cognitive Therapy and Research, 36(6), 840-846. https://doi.org/10.1007/s10608-011-9428-1

Grandchamp, R., Rapin, L., Perrone-Bertolotti, M., Pichat, C., Haldin, C., Cousin, E., Lachaux, J.-P., Dohen, M., Perrier, P., Garnier, M., Baciu, M., \& Lœvenbruck, H. (2019). The ConDialInt Model: Condensation, Dialogality, and Intentionality Dimensions of Inner Speech Within a Hierarchical Predictive Control Framework. Frontiers in Psychology, 10. https://doi.org/10.3389/fpsyg.2019.02019

Gruber, O. (2001). Effects of domain-specific interference on brain activation associated with verbal working memory task performance. Cerebral Cortex, 11(11), 1047-1055. https://doi.org/10.1093/cer cor/11.11.1047

Guimpel, B., Douilliez, C., \& Philippot, P. (2012). Validation de la version française de la mesure des dimensions de Ressassement et de Réflexion dans la Ruminative Response Scale (RRS). Communication affichée présentée dans le cadre des 41èmes Journéees Scientifiques de Thérapie Comportementale et Cognitive, Paris, France.

Hegyi, G., \& Garamszegi, L. Z. (2011). Using information theory as a substitute for stepwise regression in ecology and behavior. Behavioral Ecology and Sociobiology, 65(1), 69-76. https://doi.org/10.1007/s0 0265-010-1036-7

Hester, J. (2018). glue: Interpreted string literals. http s://CRAN.R-project.org/package=glue

Hubbard, T. L. (2010). Auditory imagery: Empirical findings. Psychological Bulletin, 136(2), 302-329. http s://doi.org/10.1037/a0018436

Hurlburt, R. T. (2011). Investigating pristine inner experience: Moments of truth. Cambridge University Press. https://doi.org/10.1017/cbo9780511842627

Hurlburt, R. T., Heavey, C. L., \& Kelsey, J. M. (2013). Toward a phenomenology of inner speaking. Consciousness and Cognition, 22(4), 1477-1494. http s://doi.org/10.1016/j.concog.2013.10.003

Iannone, R. (2018). DiagrammeR: Graph/network visualization. https://CRAN.R-project.org/package=Di agrammeR

Jacobson, E. (1931). Electrical measurements of neuromuscular states during mental activities. American Journal of Physiology, 897(1), 200-209. http s://doi.org/10.1152/ajplegacy.1931.97.1.200

Kay, M. (2018). tidybayes: Tidy data and geoms for Bayesian models. https://doi.org/10.5281/ZENODO.13 $\underline{08151}$ 
Klinger, E., \& Cox, W. M. (1987). Dimensions of thought flow in everyday life. Imagination, Cognition and Personality, 7(2), 105-128. https://doi.org/10.2190/7k 24-g343-mtqw-115v

Kruschke, J. K., \& Meredith, M. (2018). BEST: Bayesian estimation supersedes the t-test. https://CRAN.R-proje ct.org/package $=$ BEST

Lam, D., Schuck, N., Smith, N., Farmer, A., \& Checkley, S. (2003). Response style, interpersonal difficulties and social functioning in major depressive disorder. Journal of Affective Disorders, 75(3), 279-283. https://d oi.org/10.1016/s0165-0327(02)00058-7

Larsen, J. D., \& Baddeley, A. D. (2003). Disruption of verbal stm by irrelevant speech, articulatory suppression, and manual tapping: Do they have a common source? The Quarterly Journal of Experimental Psychology A: Human Experimental Psychology, 56(8), 1249-1268. https://doi.org/10.108 $\underline{0 / 02724980244000765}$

Lindblom, B. (1990). Explaining Phonetic Variation: A Sketch of the H\&H Theory. In W. J. Hardcastle \& A. Marchal (Eds.), Speech Production and Speech Modelling (pp. 403-439). Springer Netherlands. http s://doi.org/10.1007/978-94-009-2037-8_16

Livesay, J., Liebke, A., Samaras, M., \& Stanley, A. (1996). Covert speech behavior during a silent language recitation task. Perceptual and Motor Skills, 83(3), 1355-1362. https://doi.org/10.2466/pms.1996.83.3f.1 $\underline{355}$

Lœvenbruck, H., Grandchamp, R., Rapin, L., Nalborczyk, L., Dohen, M., Perrier, P., Baciu, M., \& PerroneBertolotti, M. (2018). A cognitive neuroscience view of inner language: To predict and to hear, see, feel. In P. Langland-Hassan \& A. Vicente (Eds.), Inner speech: New voices (p. 37). Oxford University Press. https://do i.org/10.1093/oso/9780198796640.003.0006

Logie, R. H., \& Baddeley, A. D. (1987). Cognitive processes in counting. Journal of Experimental Psychology: Learning, Memory, and Cognition, 13(3), 310-326. https://doi.org/10.1037/0278-7393.13.2.310

Lüdecke, D. (2018). sjstats: Statistical functions for regression models (version 0.17.1). https://doi.org/10.5 281/ZENODO.1284472

Lyubomirsky, S., Caldwell, N. D., \& Nolen-Hoeksema, S. (1998). Effects of ruminative and distracting responses to depressed mood on retrieval of autobiographical memories. Journal of Personality and Social Psychology, 75(1), 166-177. https://doi.org/10.1 037/0022-3514.75.1.166

Macken, W. J., \& Jones, D. M. (1995). Functional characteristics of the inner voice and the inner ear: Single or double agency? Journal of Experimental Psychology: Learning, Memory, and Cognition, 21(2), 436-448. https://doi.org/10.1037/0278-7393.21.2.436

Martin, L. L., \& Tesser, A. (1996). Some ruminative thoughts. In R. S. Wyer (Ed.), Advances in social cognition (Vol. 9, pp. 1-47). Lawrence Erlbaum Associates, Inc.

Mathôt, S., Schreij, D., \& Theeuwes, J. (2012). OpenSesame: An open-source, graphical experiment builder for the social sciences. Behavior Research Methods, 44(2), 314-324. https://doi.org/10.3758/s13 428-011-0168-7
McLaughlin, K. A., Borkovec, T. D., \& Sibrava, N. J. (2007). The effects of worry and rumination on affect states and cognitive activity. Behavior Therapy, 38(1), 23-38. https://doi.org/10.1016/j.beth.2006.03.003

Moberly, N. J., \& Watkins, E. R. (2008). Ruminative selffocus and negative affect: An experience sampling study. Journal of Abnormal Psychology, 117(2), 314-323. https://doi.org/10.1037/0021-843x.117.2.31 4

Morin, A. J. S., Moullec, G., Maïano, C., Layet, L., Just, J.-L., \& Ninot, G. (2011). Psychometric properties of the Center for Epidemiologic Studies Depression Scale (CES-D) in French clinical and nonclinical adults. Revue d'Épidémiologie Et de Santé Publique, 59(5), 327-340. https://doi.org/10.1016/i.respe.2011.0 $\underline{3.061}$

Nalborczyk, L., Batailler, C., Lœvenbruck, H., Vilain, A., \& Bürkner, P.-C. (2019). An introduction to Bayesian multilevel models using brms: A case study of gender effects on vowel variability in standard indonesian. Journal of Speech, Language, and Hearing Research, 62(5), 1225-1242. https://doi.org/10.1044/2018_jslhrs-18-0006

Nalborczyk, L., Perrone-Bertolotti, M., Baeyens, C., Grandchamp, R., Polosan, M., Spinelli, E., Koster, E. H. W., \& Lœvenbruck, H. (2017). Orofacial electromyographic correlates of induced verbal rumination. Biological Psychology, 127, 53-63. http s://doi.org/10.1016/j.biopsycho.2017.04.013

Nolen-Hoeksema, S., \& Morrow, J. (1993). Effects of rumination and distraction on naturally occurring depressed mood. Cognition and Emotion, 7(6), 561-570. https://doi.org/10.1080/0269993930840920 $\underline{6}$

Oppenheim, G. M., \& Dell, G. S. (2008). Inner speech slips exhibit lexical bias, but not the phonemic similarity effect. Cognition, 106(1), 528-537. https://d oi.org/10.1016/j.cognition.2007.02.006

Oppenheim, G. M., \& Dell, G. S. (2010). Motor movement matters: The flexible abstractness of inner speech. Memory \& Cognition, 38(8), 1147-1160. http s://doi.org/10.3758/mc.38.8.1147

Papageorgiou, C., \& Wells, A. (2001). Metacognitive beliefs about rumination in recurrent major depression. Cognitive and Behavioral Practice, 8(2), 160-164. https://doi.org/10.1016/s1077-7229(01)8002 $1-3$

Pedersen, T. L. (2017). Patchwork: The composer of ggplots. https://github.com/thomasp85/patchwork

Pedersen, T. L. (2018). Ggforce: Accelerating "ggplot2.” ht tps://CRAN.R-project.org/package $=$ ggforce

Perrone-Bertolotti, M., Rapin, L., Lachaux, J.-P., Baciu, M., \& Lœvenbruck, H. (2014). What is that little voice inside my head? Inner speech phenomenology, its role in cognitive performance, and its relation to selfmonitoring. Behavioural Brain Research, 261, 220-239. https://doi.org/10.1016/j.bbr.2013.12.034

R Core Team. (2018). R: A language and environment for statistical computing. R Foundation for Statistical Computing. https://www.R-project.org/ 
Radloff, L. S. (1977). The CES-D Scale: A Self-Report Depression Scale for Research in the General Population. Applied Psychological Measurement, 1(3), 385-401. https://doi.org/10.1177/0146621677001003 $\underline{06}$

Rapee, R. M. (1993). The utilisation of working memory by worry. Behaviour Research and Therapy, 31(6), 617-620. https://doi.org/10.1016/0005-7967(93)9011 4-a

Reisberg, D., Smith, J. D., Baxter, D. A., \& Sonenshine, M. (1989). "Enacted" auditory images are ambiguous; "pure" auditory images are not. The Quarterly Journal of Experimental Psychology Section A, 41(3), 619-641. https://doi.org/10.1080/14640748908402385

Saito, S. (1994). What effect can rhythmic finger tapping have on the phonological similarity effect? Memory \& Cognition, 22(2), 181-187. https://doi.org/10.3758/bf0 $\underline{3208889}$

Saito, S. (1997). When articulatory suppression does not suppress the activity of the phonological loop. British Journal of Psychology, 88(4), 565-578. https://doi.org/ 10.1111/j.2044-8295.1997.tb02658.x

Schloerke, B., Crowley, J., Cook, D., Briatte, F., Marbach, M., Thoen, E., \& Larmarange, J. (2018). GGally: Extension to "ggplot2." https://CRAN.R-project.org/pa ckage $=$ GGally

Scott, M., Yeung, H. H., Gick, B., \& Werker, J. F. (2013). Inner speech captures the perception of external speech. The Journal of the Acoustical Society of America, 133(4), EL286-EL292. https://doi.org/10.112 $1 / 1.4794932$

Sievert, C. (2018). Plotly for $r$. https://plotly-book.cpsiev ert.me

Simmons, J. P., Nelson, L. D., \& Simonsohn, U. (2012). A 21 word solution. The Official Newsletter of the Society for Personality and Social Psychology, 26(2). https://do i.org/10.2139/ssrn.2160588

Smith, J. D., Wilson, M., \& Reisberg, D. (1995). The role of subvocalization in auditory imagery. Neuropsychologia, 33(11), 1433-1454. https://doi.org/ 10.1016/0028-3932(95)00074-d

Sokolov, A. (1972). Inner speech and thought. SpringerVerlag.
Stricker, S. (1880). Studien über die Sprachvorstellungen. Braumüller.

Topolinski, S., \& Strack, F. (2009). Motormouth: Mere exposure depends on stimulus-specific motor simulations. Journal of Experimental Psychology: Learning, Memory, and Cognition, 35(2), 423-433. http s://doi.org/10.1037/a0014504

Treynor, W., Gonzalez, R., \& Nolen-Hoeksema, S. (2003). Rumination reconsidered: A psychometric analysis. Cognitive Therapy and Research, 27(3), 247-259. https://doi.org/10.1023/a:1023910315561

Wagenmakers, E.-J., Lodewyckx, T., Kuriyal, H., \& Grasman, R. (2010). Bayesian hypothesis testing for psychologists: A tutorial on the Savage-Dickey method. Cognitive Psychology, 60(3), 158-189. http s://doi.org/10.1016/j.cogpsych.2009.12.001

Warren, R. M., \& Gregory, R. L. (1958). An auditory analogue of the visual reversible figure. The American Journal of Psychology, 71(3), 612. https://doi.org/10.23 $\underline{07 / 1420267}$

Watanabe, S. (2010). Asymptotic equivalence of Bayes cross validation and widely applicable information criterion in singular learning theory. Journal of Machine Learning Research, 11, 3571-3594.

Watson, D., Clark, L. A., \& Tellegen, A. (1988). Development and validation of brief measures of positive and negative affect: The PANAS scales. Journal of Personality and Social Psychology, 54(6), 1063-1070. https://doi.org/10.1037/0022-3514.54.6.1 $\underline{063}$

Wickham, H. (2016). ggplot2: Elegant graphics for data analysis. Springer-Verlag New York. http://ggplot2.org

Wickham, H. (2017). tidyverse: Easily install and load the "tidyverse." https://CRAN.R-project.org/package=tidy verse

Xie, Y. (2015). Dynamic documents with $R$ and knitr (2nd ed.). Chapman; Hall/CRC. https://yihui.name/knitr/

Zajonc, R. B. (1968). Attitudinal effects of mere exposure. Journal of Personality and Social Psychology, 9(2, Pt.2), 1-27. https://doi.org/10.1037/h0025848 


\section{SUPPLEMENTARY MATERIALS}

\section{Peer Review History}

Download: https://collabra.scholasticahq.com/article/31051-articulatory-suppression-effects-on-induced-rumination/ attachment/78219.docx?auth_token=afAa1Nm_dWP2PWSm_xAM

\section{Supplemental Material}

Download: https://collabra.scholasticahq.com/article/31051-articulatory-suppression-effects-on-induced-rumination/ attachment/78220.pdf?auth token=afAa1Nm dWP2PWSm xAM 